\title{
Sclerosing stromal tumor of ovary: a case report and review of the literatures
}

\begin{abstract}
Sclerosing stromal tumor (SST) is a rare benign ovarian neoplasm classified as a type of sex cord-stromal tumor, accounting for approximately $2 \%$ of sex cord-stromal tumors. We present a 23year old virgin female in this article as an SST case that came with complaint of menstrual abnormalities and right lower quadrant abdominal pain then the US, MRI and pathological findings will be described.
\end{abstract}

Keywords: ovarian neoplasm, sex cord-gonadal tumors, stromal tumors
Volume 3 Issue 3 - 2016

\author{
Tahereh Ashrafganjoei,' Hossein Nikpour,'2 \\ Pooria A Nowshadi ${ }^{2}$ \\ 'Department of Obstetrics \& Gynecology, Shahid Beheshti \\ university of Medical Sciences, Iran \\ ${ }^{2}$ Department of Pathology, Kerman University of Medical \\ sciences, Iran
}

\begin{abstract}
Correspondence: Tahereh Ashrafganjoei, Associate Professor, Department of Obstetrics \& Gynecology, Emam Hosein Hospital, Shahid Beheshti University of Medical Sciences, Tehran, Iran, Tel 021734334440, 09126109963,

Email dr.ganjoei@hotmail.com
\end{abstract}

Received: September 05, 2016 | Published: December 2I, 2016

\section{Introduction}

Sclerosing stromal tumor (SST) of the ovary is a distinctive and uncommon tumor, accounting for approximately $2 \%$ of sex cordstromal tumors. Chalvardjian et al., ${ }^{1}$ first described SST as a distinct subtype among ovarian sex cord-stromal tumors in 1973, and fewer than 200 cases have been reported. ${ }^{1-4}$ They occur over a wide age range but mostly in the second and third decades with mean age of 28years. ${ }^{3}$ The most common presenting features of SSTs are abdominal mass, menstrual irregularities, pelvic pain and occasionally_precocious puberty or virilization. Five of the reported cases were among the pediatric and premenarchal age groups. ${ }^{2,4,5}$ Unusual pathological features distinguish SST from other ovarian tumors. We present a case of SST as a 23years old female then we describe radiological and pathological findings of this case and review of the related literatures.

\section{Case report}

A 23-year-old virgin female, presented with complaints of irregular menstrual periods since 9months ago and dull aching constant pain in the right lower quadrant for the past 2 months. There was no evidence of hirsutism in her general physical examination. As the patient was virgin vaginal exam could not be performed and abdominal exam was normal. Routine laboratory findings and hormonal profile including complete blood counts (CBC), Fasting blood sugar (FBS), blood urea and creatinine, TSH, PRL were within normal limits. Tumor markers including Alfa-fetoprotein $<0.5$ (reference value: $<5.8 \mathrm{IU} / \mathrm{ml}$ ), CA-125:17.2 (reference value: $<35 \mathrm{U} / \mathrm{ml}$ ) and $\beta-\mathrm{HCG}: 0 \mathrm{mIU} / \mathrm{ml}$. were checked. Ultrasonography (US) of the abdomen and pelvis revealed a right solid ovarian mass, $5 \times 5 \mathrm{~cm}$ with heterogeneous echotexture. Magnetic resonance imaging (MRI) of the pelvis revealed a well defined contour mixed signal mass measuring $50 \mathrm{~mm}$ in diameter in right anterolateral portion of pelvis in favor of ovarian mass and less probably a pedunculated myoma (Figure 1). With a pre-operative diagnosis of right ovarian tumor, the patient underwent exploratory laparatomy which revealed normal abdominal and pelvic cavity without any ascites. The right ovary was occupied by a tumor, which was predominantly solid, pale yellow and homogeneous. Mass resection was performed and the specimen was sent for frozen section evaluations which were reported as a benign ovarian mass. So right ovary was preserved and surgical operation was ended. Pathologic evaluation revealed alternative hypo and hyper cellular areas composed of spindle shape cells with marked vascular spaces within the stroma. Foci of lipid-containing round to spindle cells are also noted. In follow up visits, the patient had normal mensural bleeding since twelve months after surgery.
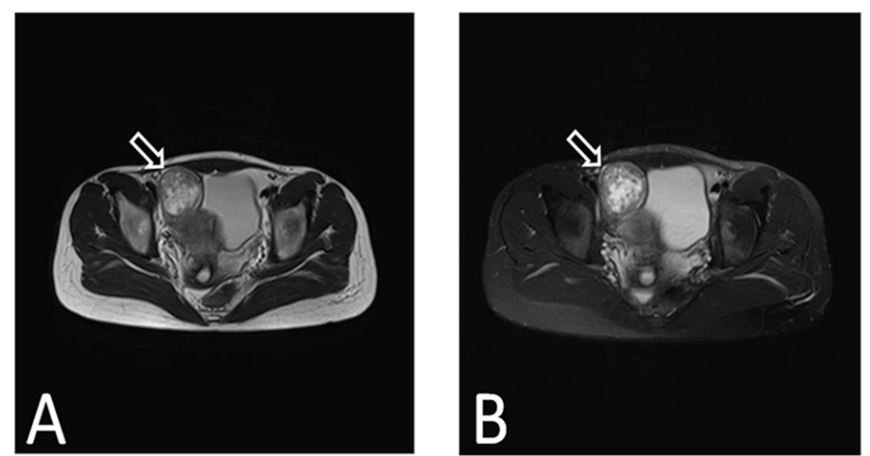

Figure I MRI imaging A TI-W \& B T2-W Axial show a well defined heterogeneous mass with high signal portion (multiloculated small cystic component) in central part of it, in right adnexa without adjacent free fluid.

\section{Discussion}

Sclerosing stromal tumor (SST) of the ovary is an extremely rare and benign subtype of a sex cord stromal tumor of the ovarian neoplasms that occur early in life $(80 \%$ occurring in second and third decades), in contrast to other stromal tumors that commonly occur during the fifth and sixth decades. Just five reported cases were among the premenstrual age range. ${ }^{5}$ The most common presenting features of SSTs are abdominal mass and menstrual irregularities 
such as metrorrhagia or postmenoposoual bleeding in elderly patients. Other symptoms are pelvic pain, palpable mass, precocious puberty (associated with secretion of estrogen and progesterone., ${ }^{2,6}$ Hormonal effects such as masculinization are uncommon. ${ }^{7}$ Sclerosing stromal tumours are rarely seen together with pregnancies; only 11 pregnant patients have been reported in literature. ${ }^{8}$ The diameter of these tumors is variable ( 3.5 to $43 \mathrm{~cm}){ }^{5-9}$ Most of the tumors are unilateral, solid and cystic and grey-white in appearance. ${ }^{10}$ There is only 3 reported case of bilateral SST. ${ }^{11}$ Mariela et al., ${ }^{12}$ showed that majority of SSTs are in right ovary as our case. ${ }^{12}$ Most SSTs are considered nonfunctioning tumors of the ovary, but some reports have described endocrine alterations caused by estrogen, progesterone and testosterone. ${ }^{1,2}$ Also including some from pregnant patients who were virilized presumably because high HCG levels during pregnancy stimulated the luteinized cells, a feature of this neoplasm, to produce androgens. ${ }^{7,8}$ Ultrasound study is a useful imaging to diagnose STT, because it usually appears as a solid lesion but could be cystic and multilocular with increased peripheral vascularity on color Doppler leading to an erroneous impression of malignancy and unnecessary extensive surgical intervention on young women affecting their fertility. ${ }^{13}$ On MR imaging an SST emerges as a heterogeneous solid mass with hyper intense cystic components or with a peripheral hypo intense rim on T2-weighted images as in the current case. Although dynamic contrast-enhanced images were not performed for the patient, the use of contrast-enhanced CT and or MR imaging for SSTs show marked early peripheral enhancement with centripetal progression and internal cystic changes. These findings are crucial in differentiation of SST from other stromal tumors, such as fibroma or the coma, that show slow and prolonged enhancement entirely, and from most of the malignant ovarian neoplasms, in which early enhancement and early washout are usually observed. ${ }^{14}$ The histological diagnosis of SST is based on three features: pseudolobular pattern with characteristic alternating hypo and hyper cellular areas, finally ectatic branching (staghorn) vessels which when prominent and aggregated may impart a hemangiopericytoma like appearance and a heterogeneous cell population of both vacuolated or luteinized cells and spindle fibroblast-like cells with intervening collagenization or sclerosis. ${ }^{9}$ Rarely, the tumors show massive sclerosis. ${ }^{15}$

The Immunohistochemical staining are positive for inhibin, calretinin, smooth muscle actin (SMA), CD 99, vimentin but negative for S-100 protein, cytokeratinand epithelialmarkers. The main differential diagnosis is with the coma if grossly uniformly solid, although in general SST have a more variegated appearance, fibroma and particularly myxoma are the two other tumors witch may be mimic the STT histologic patterns. But pseudolobulation and prominent vasculature are extremely rare in luteinized thecomasand fibromas. ${ }^{15}$ The excised tumor of the patient reported here showed hypo and hyper cellular areas composed of spindle shape cells with marked vascular spaces within the stroma. Immunohistochemical studies were positive for SMA and Desmin. Surgical resection of the tumor is the primary treatment modality. In the presence of unilateral SST, there is no concord with the available literature that biopsy would be indicated for a normal appearing contralateral ovary, given the rarity of bilateral SST. It usually has excellent prognosis with dramatic reversal of symptoms.

\section{Conclusion}

Due to the rarity of this particular ovarian neoplasm it should be considered in young women with menstrual irregularity, and unilateral complex adnexal masses. Frozen biopsies should be performed during surgery. Although this definite diagnosis is based on pathology postoperatively, so a number of issues that should be considered in the assessment these patients including proper preoperative evaluation such as imaging and tumour markers and hormonal profile evaluation. So accurate preoperative evaluation and intraoperative pathologic assessment help to prevent the resection of the normal part of the ovary.

\section{Acknowledgements}

None.

\section{Conflict of interest}

The author declares no conflict of interest.

\section{References}

1. Chalvardjian A, Scully RE. Sclerosing stromal tumors of the ovary. Cancer. 1973;31(3):664-670.

2. Chang W, Oiseth SJ, Orentlicher R, et al. Bilateral sclerosing stromal tumor of the ovaries in a premenarchal girl. Gynecol Oncol. 2006;101(2):342-345.

3. Zhai T, Cui M, Chen G, et al. Sclerosing stromal ovarian tumor combined with early onset severe preeclampsia. a case report. J Reprod Med. 2015;60(5-6):249-253.

4. Ozdemir O, Sari ME, Sen E, et al. Sclerosing stromal tumour of the ovary: A case report and the review of literature. Niger Med J. 2014;55(5):432-437.

5. He Y, Yang KX, Jiang W, et al. Sclerosing stromal tumor of the ovary in a 4-year-old girl with characteristics of an ovarian signet-ring stromal tumor. Pathol Res Pract. 2010;206(5):338-341.

6. Chang YW, Hong SS, Jeen YM, et al. Bilateral sclerosing stromal tumor of the ovary in a premenarchal girl. Pediatr Radiol. 2009;39:731-734.

7. Park SM, Kim YN, Woo YJ, et al. A sclerosing stromal tumor of the ovary with masculinization in a premenarchal girl. Korean J Pediatr. 2011;54(5):224-227.

8. Kaygusuz EI, Cesur S, Cetiner H, et al. Sclerosing stromal tumour in young women: clinicopathologic and immunohistochemical spectrum. J Clin Diagn Res. 2013;7(9):1932-1935.

9. Youm HS, Cha DS, Han KH, et al. A case of huge sclerosing stromal tumor of the ovary weighing $10 \mathrm{~kg}$ in a 71-year-old postmenopausal woman. J Gynecol Oncol. 2008;19(4):270-274.

10. Nucci MR, Lee KR, Crum CP. Sex cord-stromal and miscellaneous tumors of the ovary. Diagnostic Gynecologic and Obstetric Pathology. USA: Elsevier Saunders; 2006. p. 1-971.

11. Naidu A, Chung B, Simon M, et al. Bilateral sclerosing stromal ovarian tumor in an adolescent. Case Rep Radiol. 2015;2015:271394.

12. Marelli G, Carinelli S, Mariani A, et al. Sclerosing stromal tumor of the ovary. Report of eight cases and review of the literature. Eur J Obstet Gynecol Reprod Biol. 1998;76(1):85-89.

13. Deval B, Rafii A, Darai E, et al. Sclerosing stromal tumor of the ovary: color doppler findings. Ultrasound Obstet Gynecol. 2003;22(5):531534.

14. Matsubayashi R, Matsuo Y, Doi J, et al. Sclerosing stromal tumor of the ovary: radiologic findings. Euro Radiol. 1999;9(7):1335-1338.

15. Esther O, Robert HY. Stromal tumours of the ovary: an update. Diagnostic Histology. 2014;20(9):376-384. 